\title{
Expression of GOLPH3 in patients with non-small cell lung cancer and xenografts models
}

\author{
SHICONG TANG ${ }^{1 *}$, RIRONG YANG $^{2 *}$, XIN ZHOU $^{3}$, HONG PAN $^{3}$ and JIANLUN LIU ${ }^{1}$ \\ ${ }^{1}$ Department of Breast Surgery, The Affiliated Tumor Hospital of Guangxi Medical University; ${ }^{2}$ Department of Immunology, \\ School of Preclinical Medicine, Guangxi Medical University; ${ }^{3}$ Department of Thoracic Surgery, \\ The Affiliated Tumor Hospital of Guangxi Medical University, Nanning, Guangxi 530021, P.R. China
}

Received March 4, 2017; Accepted October 26, 2017

DOI: $10.3892 / \mathrm{ol} .2018 .8340$

\begin{abstract}
Increased expression of Golgi phosphoprotein 3 (GOLPH3) has been reported to be associated with several types of human cancer. Patient-derived cancer xenograft models have demonstrated great potential in preclinical studies. In the present study, the link between GOLPH3 expression and survival was examined in patients with non-small cell lung cancer (NSCLC). Patient-derived lung cancer xenograft models were established with two different methods. Lastly, the association between GOLPH3 expression and establishment of the xenograft models was explored. Reverse transcription-quantitative polymerase chain reaction (RT-qPCR) and immunohistochemistry analysis were used to examine GOLPH3 expression in 60 NSCLC tissues and matched adjacent non-cancerous tissues (ANT). In addition, tumor pieces from the 60 NSCLC tissues were implanted in the subcutaneous layer and in the subrenal kidney capsule of nude mice. RT-qPCR, histopathology and immunohistochemistry were used to confirm the human origin of the xenograft tumors. RT-qPCR was also used to research the mutation status of GOLPH3 in the xenograft tumors. The results demonstrated that NSCLC tissues had higher expression of GOLPH3, at the mRNA and protein level, compared with ANT. High expression of GOLPH3 correlated with poor survival in patients with NSCLC. Successful engraftment was established for 27 tissues in the subrenal kidney capsule and
\end{abstract}

Correspondence to: Professor Jianlun Liu, Department of Breast Surgery, The Affiliated Tumor Hospital of Guangxi Medical University, 71 Hedi Road, Nanning, Guangxi 530021, P.R. China E-mail: liu_jianlun@126.com

Professor Hong Pan, Department of Thoracic Surgery, The Affiliated Tumor Hospital of Guangxi Medical University, 71 Hedi Road, Nanning, Guangxi 530021, P.R. China

E-mail: pan_hong2017@126.com

*Contributed equally

Key words: non-small cell lung cancer, Golgi phosphoprotein 3, xenograft models for 16 in the subcutaneous layer of nude mice. The subrenal kidney capsule group demonstrated significantly higher engraftment rates than the subcutaneous layer group. In addition, higher GOLPH3 expression in the tumor tissues was significantly correlated with higher engraftment rates in mice. In both groups, few xenografts lost the GOLPH3 mutation. In summary, GOLPH3 may be an important diagnosis and prognosis indicator in patients with NSCLC. The genotype and phenotype of the xenograft tumors derived from patient lung cancer tissues exhibited significant similarities to the originating primary tumors. High GOLPH3 expression may promote the successful establishment of xenograft models for NSCLC.

\section{Introduction}

Recent studies have reported that lung cancer imposes a severe impact on human health. In numerous countries, the mortality from lung cancer is still increasing $(1,2)$. Non-small cell lung cancer (NSCLC) accounts for 70-80\% of all lung cancer cases (3). It is therefore paramount to identify new therapy targets and methods targeting NSCLC.

Golgi phosphoprotein 3 (GOLPH3), also known as GPP34 or GMx33, is a newly identified 34-kDa protein in the trans-Golgi matrix, which serves a role in anterograde and retrograde Golgi trafficking (4-6). Previous studies have demonstrated that GOLPH3 exhibits a significant association with cancer (7-9). Multiple studies have reported that overexpression of GOLPH3 is associated with poor survival in patients with cancer (10-15). High expression of GOLPH3 is linked to high proliferation and migration potential in cancer cells $(10,11,14,15)$. In addition, high expression of GOLPHP3 is correlated with neoadjuvant chemotherapy resistance (13). Nevertheless, the expression of GOLPH3 and its role in the establishment of xenograft models remain largely unknown.

A number of studies have now reported that animal tumor xenograft models can serve as excellent tools in predicting efficacy and toxicities for cancer chemotherapeutics prior to entering the clinic (16-18).Immunodeficient mice are considered an ideal host for establishing xenografts. Patient-derived cancer xenograft models provide several advantages. First, xenograft models offer the preservation of original tumor tissue histology, by maintaining the original tumor stroma, which 
is essential for tumor growth (19). Secondly, the xenograft model can provide a continuous source of live, transplantable tumor tissues, starting from only minute amounts of biopsy material and surgical specimens (17). The third advantage of xenografts models lies in their ability to recapitulate the general phenotypic and genotypic characteristics of the tumors from which they originated $(17,20,21)$. However, there are also several limitations to xenografts models. A main limitation is that small samples are used to establish the grafts, and therefore the entire heterogeneous tumor may not be well represented in the final xenograft. Several studies have therefore suggested using multiple samples from the same tumor in order to potentially address this limitation $(17,22)$.

Rygaard and Povlsen (23) successfully transplanted human lung cancer tissues into the subcutaneous layer of nude mice for the first time in 1969. Cutz et al (17) reported results for grafted tumor tissues in the mouse kidney capsule and achieved engraftment rates of $>90 \%$ (17). Fichtner et al (24) collected fragments from 102 NSCLS tissues and grafted these in the subcutaneous layer of NOD/Scid mice to establish xenograft models and reported a take rate of 24.5\%. Perez-Soler et al (25) used the same method as Fichtner et al (24) to establish the xenograft models and reported engraftment rates of $34 \%$. A number of scholars support that xenografts in the kidney capsule attained a higher engraftment rate than in the subcutaneous layer.

In the present study, GOLPH3 expression in NSCLC tissues and its link to survival of patients with NSCLC were examined. Then, surgically resected NSCLC samples were obtained and transplanted into the subcutaneous layer and the subrenal kidney capsule of immunodeficient mice, with the aim to establish patient-derived lung cancer xenograft models, to examine the most efficient method of engraftment, and to explore the association between GOLPH3 expression and the establishment of xenograft models.

\section{Materials and methods}

Patients, tissue samples and experimental animals. Matched pairs of cancerous tissues and adjacent non-cancerous tissues (ANT) were obtained from 60 patients with NSCLC at the Department of Thoracic Surgery, The Affiliated Tumor Hospital of Guangxi Medical University (Nanning, China) from January 1, 2011 to December 31, 2011. Follow-up of the patients was recorded from January 1, 2012 to December 31, 2016. The specimens were fixed in $10 \%$ formalin and embedded in paraffin, following which $3 \mu \mathrm{m}$ sections were prepared for pathological analysis. Tumor pathology was analyzed for all specimens by the same hospital pathologist. The protocols of the present study were approved by the Ethics Committee of Tumor Hospital of Guangxi Medical University (Nanning, China). Prior to collecting samples of NSCLC and ANT, written informed consent was acquired from all enrolled patients. A total of 120 nude mice (age, 3-5 weeks; sex, female; weight, 18-22 g), obtained from the Guangxi Laboratory Animal Center of Guangxi Medical University (Nanning, China), were used to establish the xenograft models in the present study. All animals were maintained in specific pathogen-free environment at $25-27^{\circ} \mathrm{C}$ and with 25-50\% humidity. The animal experiments obeyed ARRIVE
Guidelines and AVMA Guidelines for the Euthanasia of Animals 2013 Edition (26,27).

Establishment of xenograft models. Tumor tissue samples were obtained and divided into pieces of $\sim 2 \times 3 \times 3 \mathrm{~mm}$ under sterile conditions, stored in RPMI-1640 medium (Gibco; Thermo Fisher Scientific, Inc., Waltham, MA, USA) without dimethyl sulfoxide and incubated in an icebox for later implantation. The duration between tumor tissue harvest and implantation into nude mice was $<30 \mathrm{~min}$. Nude mice were anesthetized by intraperitoneal injection of Avertin $(250 \mathrm{mg} / \mathrm{kg}$; Tianjin Kermal Chemical Reagent Co., Ltd., Tianjin, China). The frozen tumor tissues were thawed at $37^{\circ} \mathrm{C}$.

For the kidney capsule engraftment, a $1 \mathrm{~cm}$ incision along the dorsal skin midline of the mouse, along with another incision on the body wall slightly shorter than the kidney's long axis, was made. The kidney was slipped out of the body cavity using forefinger and thumb to press the other side of the organ. A micro-operation was conducted on the kidney capsule to make a $2 \mathrm{~mm}$ incision and insert 2-3 tumor pieces into the surface of the kidney using a polished glass pipette through the incision. To avoid bleeding, it is important not to damage the kidney parenchyma. The kidney capsule incision was then sutured, and the kidney was gently eased back into the body cavity. All other incisions of skin and body wall were sutured.

For the subcutaneous engraftment, a $1 \mathrm{~cm}$ incision along the dorsal skin midline of the nude mouse was made and the thawed tumor tissues (1-2 pieces) were implanted subcutaneously using a trocar needle. Following the operation, the skin incision was gently sutured.

Two or three mice were housed per cage with plenty of food and water and their health was observed daily. The graft tumors were monitored daily. For the subcutaneous group, the long and short axes of tumors were measured once a week using a caliper, in order to calculate their volume and growth rate. The humane endpoint was set at tumors with a volume of $500 \mathrm{~mm}^{3}$; following this, mice were euthanized and grafts were collected The harvested tumors were separated into two portions: one portion was snap frozen and stored in liquid nitrogen; the other portion was fixed in formalin and embedded in paraffin for histological analysis.

Reverse transcription-quantitative polymerase chain reaction $(R T$ - $q P C R)$. RNA was extracted from both primary lung cancer tissues and tumors harvested from xenograft mice using the MiniBEST Universal RNA Extraction kit (Takara Biotechnology Co., Ltd., Dalian, China) according to the manufacturer's protocol. RNA from frozen primary cancer tissues was extracted using the aforementioned kit (Takara Biotechnology Co., Ltd.). The concentration of total RNA was measured on a Peqlab NanoDrop (Thermo Fisher Scientific, Inc.) and the quality was examined using RNA LabChips on the Agilent Bioanalyzer 2100 (Agilent Technologies Inc., Santa Clara, CA, USA). RNA with an integrity index $>6.5$ was used for cDNA synthesis. cDNA was generated using PrimeScript RT kit (Takara Biotechnology Co., Ltd.). The GOLPH3 primers were as follows: Forward, 5'-GGGCGA CTCCAAGGAAAC-3' and reverse, 5'-CAGCCACGTAAT CCAGATGAT-3'. Ki67 primers were as follows: Forward, 5'-GACCTGTTCTTTGAGGCTGAC-3' and reverse, 5'-TCC 
ATCTTCTTCTTTGGGTATTGTT-3'. Mitochondria primers were as follows: Forward, 5'-TGTCTTCCTCACCGATTC CT-3' and reverse, 5'-ACCACCCGAGCTCTGTCTTACTC-3'. $\beta$-actin was used as the internal control with specific primers: Forward, 5'-GCACCGTCAAGGCTGAGAAC-3' and reverse, 5'-TGGTGAAGACGCCAGTGGA-3'. qPCR was performed using SYBR Premix Ex Taq II (Takara Biotechnology Co., Ltd.), and reactions were run in duplicates at $95^{\circ} \mathrm{C}$ for $30 \mathrm{sec}$ for 1 cycle, $95^{\circ} \mathrm{C}$ for $3 \mathrm{sec}$ and $60^{\circ} \mathrm{C}$ for $30 \mathrm{sec}$ for 40 cycles. The $\mathrm{C}_{\mathrm{q}}$ values of the samples were calculated, and the relative mRNA levels were analyzed using the $2^{-\Delta \Delta \mathrm{Cq}}$ method (24). Mutational analysis of GOLPH3 was performed using a set of primer pairs: Forward, 5'-GAGCGTTTCTGCTTTGGGAC-3' and reverse, 5'-TGTGCTTTGGCAATTCTGGTG-3', that covered the entire coding region of the GOLPH3 gene. All fragments were sequenced using the BigDye Terminator Cycle Sequencing kit and ABI3730 automated sequencer (Applied Biosystems; Thermo Fisher Scientific, Inc.). Each mutation was confirmed in duplicate.

Histopathology and immunohistochemistry. Mice were euthanized and xenograft tumors were excised and recorded for size, shape and histological characteristics. The original lung cancer specimens and their xenografts were fixed in $10 \%$ formalin for $24 \mathrm{~h}$, and embedded into paraffin. Paraffin sections of $4 \mu \mathrm{m}$ were cut and mounted on glass slides. Routine hematoxylin and eosin staining was performed for histopathological analysis (28). GOLPH3 expression was assessed by evaluating the proportion and intensity of positively stained carcinoma cells. A score was assigned to represent the estimated percentage of positively stained carcinoma cells as follows: 0 , none; $1, \leq 50 \% ; 2,50-75 \%$; and $3, \geq 75 \%$. An intensity score was assigned to represent the average estimated intensity of staining in positive carcinoma cells as follows: 0 , none; 1 , weak; 2 , intermediate; and 3 , strong. The proportion score and intensity score were multiplied to obtain a total score ranging from 0 to 9 . The immunohistochemistry results were classified based on total scores, with 0-4 classified as +, 5-6 indicating ++, and 7-9 indicating +++. Those of + and ++ were classified as low expression, and +++ was regarded as high expression (13).

Three antibodies were used for immunohistochemistry analysis purchased from Abcam, Cambridge, UK; anti-mitochondria (cat. no. ab92824; 1:100 dilution), anti-GOLPH3 (cat. no. ab91492; 1:150 dilution) and anti-Ki-67 (cat. no. ab15580; 1:200 dilution). Sections were incubated for $1 \mathrm{~h}$ at $60^{\circ} \mathrm{C}$ and dewaxed for $40 \mathrm{~min}$ in dimethylbenzene, and serially hydrated in 100, 95, 80 and $75 \%$ alcohol solutions in distilled water. Antigen retrieval was performed using citrate antigen retrieval solution (Beyotime Institute of Biotechnology, Guangzhou, China) at $100^{\circ} \mathrm{C}$ for $5 \mathrm{~min}$, then sections were cooled to room temperature and washed with PBS for $3 \mathrm{~min}$. Following washing with PBS, the sections were blocked with 5\% normal goat serum (Gibco; Thermo Fisher Scientific, Inc.) in PBS for $30 \mathrm{~min}$ at room temperature. To quench endogenous peroxidase activity, slides were incubated with $0.5 \%$ hydrogen peroxide in methanol for $30 \mathrm{~min}$. Primary antibodies were added to the sections and incubated overnight at $4{ }^{\circ} \mathrm{C}$. The sections were then rewarmed for $30 \mathrm{~min}$ at room temperature, washed with PBS three times for $5 \mathrm{~min}$, and incubated with the following secondary antibodies; horseradish peroxidase (HRP) rabbit anti-mouse (cat. no. ab97046; 1:200) and HRP goat anti-rabbit (cat. no. ab97051; 1:200; both Abcam) for $30 \mathrm{~min}$ at $37^{\circ} \mathrm{C}$. Following three additional $5 \mathrm{~min}$ washes with PBS, $0.05 \%$ 3,3' diaminobenzidine was added for signal detection. Sections were serially dehydrated in $100,95,80$, and $75 \%$ graded alcohols and counterstained with hematoxylin.

Statistical analysis. Statistical analyses were performed using SPSS 22.0 (IBM Corp., Armonk, NY, USA). The association between expression of GOLPH3 and clinicopathological features was analyzed using $\chi^{2}$ tests, while a Fisher's exact test was used when the unit was $<6$. The log-rank test and Kaplan-Meier estimator method were used for survival analyses. Cox proportional hazard model was used for univariate and multivariate analyses. One-way analysis of variance (ANOVA) tests were used for the comparison between gene expression and internal reference expression. A post-hoc Bonferroni test was applied following ANOVA when the results showed statistical significance. $P<0.05$ was considered to indicate a statistically significant difference.

\section{Results}

Expression of GOLPH3 in NSCLC tissues. RT-qPCR analysis was performed to determine the expression of GOLPH3 in 60 matched pairs of NSCLC tissues and ANT. The results revealed that, compared with ANT, NSCLC tissues exhibited significantly increased levels of GOLPH3 mRNA expression $(\mathrm{P}<0.001$; Fig. 1A). Results from immunohistochemistry analysis also demonstrated higher expression of GOLPH3 protein in tumor tissues compared with ANT (Fig. 1B). According to the results from the immunohistochemistry analysis, and the level of positive staining, the expression of GOLPH3 protein in the tissues was further classified into low and high expression (Fig. 1C).

Association between GOLPH3 expression and clinicopathological features of NSCLC patients. The association between GOLPH3 expression and the clinicopathological features of patients with NSCLC are presented in Table I. GOLPH3 expression level was significantly associated with lymph node metastasis $(\mathrm{P}=0.005)$ and distant metastasis $(\mathrm{P}=0.002)$. Univariate and multivariate analyses indicated that lymph node metastasis, distant metastasis and expression of GOLPH3 were independent prognostic factors (Table II). The results of log-rank test survival analysis exhibited that the patients with increased expression of GOLPH3 displayed a significantly decreased overall survival compared with patients with low expression of GOLPH3 (P<0.001; Fig. 1D).

Establishment of patient-derivedxenografts. Tissue specimens obtained from 60 lung cancer patients enrolled in the present study were implanted into nude mice, using two different methods: engraftment into the subcutaneous layer and into the subrenal kidney capsule. Successful engraftment was established for 27 tissues in the subrenal kidney capsule group and for 16 tissues in the subcutaneous layer group (Table III). The engraftment rates were therefore $45 \%$ for the subrenal kidney capsule group and $26.67 \%$ for the subcutaneous layer 
A

GOLPH3 expression in NSCLC and ANT

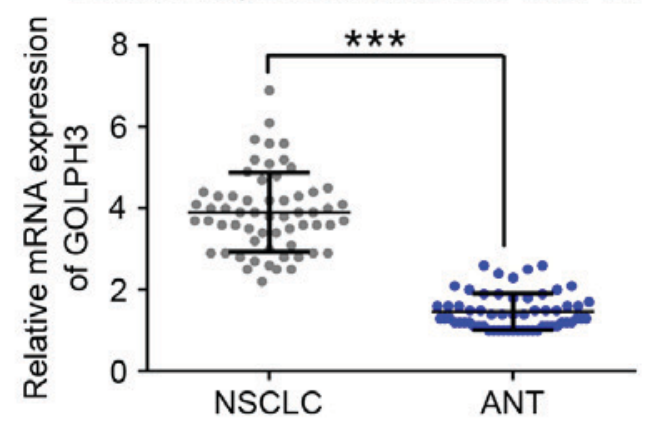

C

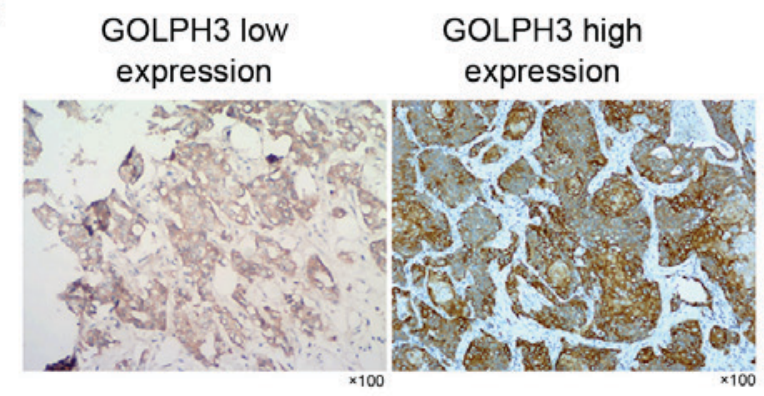

B
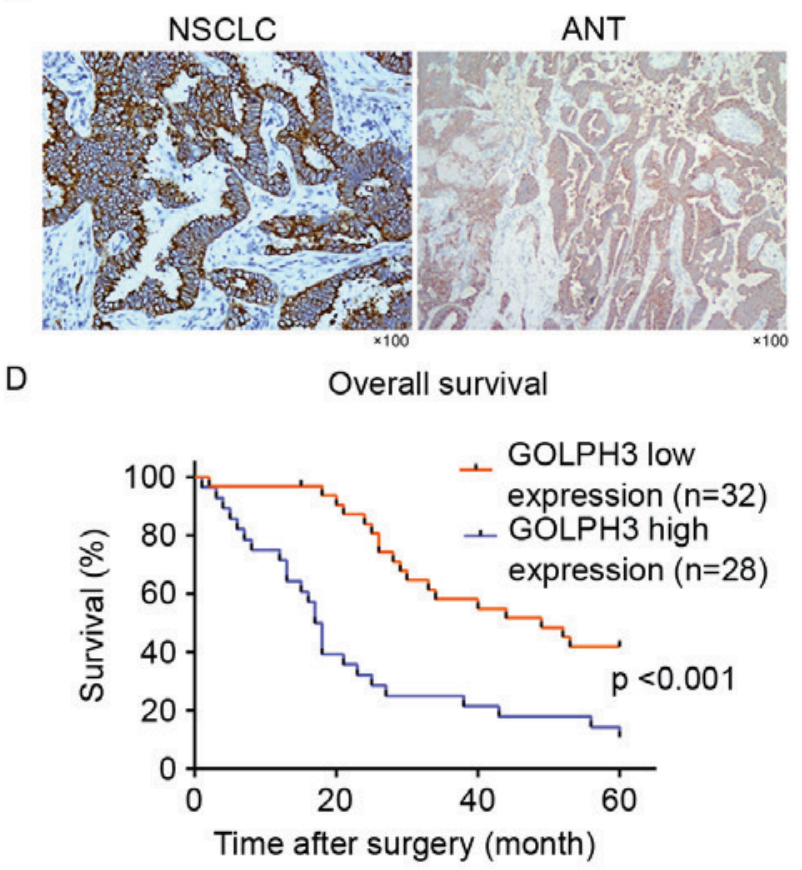

Figure 1. GOLPH3 expression in NSCLC. (A) mRNA expression of GOLPH3 in NSCLC tissues and ANT from 60 patients. ${ }^{* * *}$ P $<0.001$. (B) Representative images from immunohistochemistry analysis of NSCLC tissues and ANT for the protein expression of GOLPH3 (magnification, x100). (C) Representative images of tissues with low and high GOLPH3 expression (magnification, x100). (D) High expression of GOLPH3 is significantly correlated with poor overall survival in patients with NSCLC. GOLPH3, Golgi phosphoprotein 3; NSCLC, non-small cell lung cancer; ANT, adjacent non-cancerous tissue.

group. The subrenal kidney capsule group demonstrated a significantly higher engraftment rate compared with the subcutaneous layer group ( $\mathrm{P}=0.036$; Table III). To confirm the human origin of the xenograft tumors, qPCR assays were preformed to analyze Ki-67 and mitochondria expression with human and mouse-specific primers. The results demonstrated that the xenografts exhibited higher expression of human Ki-67 and mitochondria compared with their murine homologs (Fig. 2A). The results were confirmed by an one-way ANOVA test, $\mathrm{P}<0.05$. The histopathology and immunohistochemistry analysis also demonstrated that xenografts derived from a human squamous cell carcinoma and an adenocarcinoma exhibited positive signal for human-specific antibodies and phenotypic similarity to the original patient-derived tissues (Fig. 2B and C).

Association between GOLPH3 expression and xenograft engraftment. The 27 successful xenografts from the subrenal kidney capsule group were further analyzed. When the original tissues were examined for these xenografts, 18 exhibited high expression of GOLPH3 and 9 exhibited low expression. The results of $\chi^{2}$ tests demonstrated that, compared with decreased GOLHP3 expression, tumors with increased GOLPH3 expression displayed higher engraftment rates (Table IV; $\mathrm{P}=0.005$ ). Next, the 16 successful xenografts of the subcutaneous layer group were analyzed. When the original tissues were examined for these xenografts, 10 exhibited high expression and 6 low expression, and high GOLPH3 expression was significantly associated with engraftment rate (Table IV; $\mathrm{P}=0.036$ ), similar to the subrenal kidney capsule group. Finally, qPCR was used to examine whether xenografts successfully recapitulated the
GOLPH3 genotype of the original tumors. In the subrenal kidney capsule group, only 2/9 and 3/18 in the GOLPH3 lowand high-expression tumors, respectively, lost the mutation $(\mathrm{P}=1$; Table $\mathrm{V})$. In the subcutaneous layer group, only $2 / 6$ and 2/10 in GOLPH3 low- and high-expression tumors, respectively, lost the mutation (Table V; $\mathrm{P}=0.604$ ). These results suggested that, independent of GOLPH3 expression levels, the mutation status of the GOLPH3 gene in the patient tumors was generally preserved in the xenograft tumors. Overall, the patient-derived NSCLC xenograft models were demonstrated to carry the human genotype and phenotype accurately.

\section{Discussion}

In the present study, GOLPH3 was demonstrated to be highly expressed in a cohort of NSCLC tissues, indicating that GOLPH3 may be a useful diagnostic factor for NSCLC. In addition, it was demonstrated that GOLPH3 expression was significantly correlated with poor overall survival, suggesting that GOLPH3 may play a significant role as a prognosis indicator in patients with NSCLC.

The present study is the first report of GOLPH3 expression and mutation status in the establishment of xenograft models. Novel patient-derived lung cancer xenograft models were established and the engraftment rates were compared using two different methods: subcutaneous layer and subrenal kidney capsule engraftment. The xenograft tumors, as well as the original tumors they were derived from, were assessed by qPCR and immunohistochemistry, and the results demonstrated that the xenografts preserved most of the original genotypic and phenotypic characteristics of the original 
Table I. Association between GOLPH3 expression and the clinicopathological features of 60 patients with non-small cell lung cancer.

\begin{tabular}{|c|c|c|c|c|}
\hline \multirow[b]{2}{*}{ Variables } & \multirow[b]{2}{*}{ Number of patients } & \multicolumn{2}{|c|}{ Expression of GOLPH3 } & \multirow[b]{2}{*}{ P-value } \\
\hline & & Low $(n=32)$ & High $(n=28)$ & \\
\hline Age, years & & & & 0.128 \\
\hline$<60$ & 32 & 20 & 12 & \\
\hline$\geq 60$ & 28 & 12 & 16 & \\
\hline Tumor invasion depth, cm & & & & 0.464 \\
\hline$<5$ & 20 & 12 & 8 & \\
\hline$\geq 5$ & 40 & 20 & 20 & \\
\hline Lymph node metastasis & & & & 0.005 \\
\hline With & 43 & 18 & 25 & \\
\hline Without & 17 & 14 & 3 & \\
\hline Distant metastasis & & & & 0.002 \\
\hline With & 24 & 7 & 17 & \\
\hline Without & 36 & 25 & 11 & \\
\hline Pathological type & & & & 0.796 \\
\hline Adenocarcinoma & 30 & 16 & 14 & \\
\hline Squamous cell carcinoma & 30 & 15 & 15 & \\
\hline Sex & & & & 0.267 \\
\hline Male & 43 & 21 & 22 & \\
\hline Female & 17 & 11 & 6 & \\
\hline
\end{tabular}

GOLPH3, Golgi phosphoprotein 3.

Table II. Cox regression analysis for overall survival.

\begin{tabular}{|c|c|c|c|c|c|c|}
\hline \multirow[b]{2}{*}{ Variables } & \multicolumn{3}{|c|}{ Univariate analysis } & \multicolumn{3}{|c|}{ Multivariate analysis } \\
\hline & $\begin{array}{l}\text { Hazard } \\
\text { ratio }\end{array}$ & $\begin{array}{l}95 \% \text { confidence } \\
\text { interval }\end{array}$ & P-value & $\begin{array}{l}\text { Hazard } \\
\text { ratio }\end{array}$ & $\begin{array}{c}95 \% \text { confidence } \\
\text { interval }\end{array}$ & P-value \\
\hline Age & 0.999 & $0.965-1.034$ & 0.953 & 0.987 & $0.952-1.023$ & 0.476 \\
\hline Tumor invasion depth & 1.187 & $0.656-2.146$ & 0.571 & 0.737 & $0.466-1.716$ & 0.737 \\
\hline Lymph node metastasis & 2.451 & $0.650-2.871$ & 0.012 & 1.635 & $0.838-1.822$ & 0.046 \\
\hline Distant metastasis & 2.281 & $0.731-2.458$ & 0.025 & 1.739 & $0.607-2.140$ & 0.038 \\
\hline Pathological type & 0.728 & $0.399-1.330$ & 0.302 & 0.583 & $0.279-1.221$ & 0.152 \\
\hline Sex & 0.999 & $0.965-1.034$ & 0.953 & 1.242 & $0.542-2.846$ & 0.609 \\
\hline GOLPH3 expression & 1.628 & $1.205-2.200$ & 0.001 & 1.705 & $1.247-2.332$ & 0.003 \\
\hline
\end{tabular}

GOLPH3, Golgi phosphoprotein 3.

tumors. Human-specific markers for Ki-67 and mitochondria were expressed normally in the primary lung cancer. When the xenograft tumors were analyzed, the results demonstrated that the human-specific Ki-67 and mitochondria markers were expressed higher than the murine. These results confirmed that the xenograft tumors were of human origin. The murine genes were expressed at lower levels, due to the xenograft stroma consisting of both human cancer stroma from the original tumors and murine stroma from the mouse host. However, the human cancer stroma was more predominant than that of the nude mice. The morphological characteristics analyzed by immunohistochemistry also revealed that the xenografts were originated from the human primary lung cancer tissue.

The present study is the first to directly compare the engraftment rates in subcutaneous layer method and subrenal kidney capsule method. Both methods can establish xenograft 
Table III. Comparison of engraftment rates between the subcutaneous layer and the subrenal kidney capsule methods.

\begin{tabular}{lcccr}
\hline Engraftment method & Total (n) & Success (n) & Failure (n) & P-value \\
\hline Subcutaneous layer & 60 & 16 & 44 & 0.036 \\
Subrenal kidney capsule & 60 & 27 & 33 &
\end{tabular}

Table IV. Association between GOLPH3 expression and engraftment rates.

\begin{tabular}{lccccccc}
\hline & \multicolumn{3}{c}{ Subrenal kidney capsule } & & \multicolumn{3}{c}{ Subcutaneous layer } \\
\cline { 2 - 3 } GOLPH3 expression & Success (n) & Failure (n) & P-value & & Success (n) & Failure (n) & P-value \\
\hline Low & 9 & 23 & 0.005 & & 6 & 36 & 0.036 \\
High & 18 & 10 & & & 10 & 18 & \\
\hline
\end{tabular}

GOLPH3, Golgi phosphoprotein 3.

Table V. GOLPH3 expression and mutation status in xenograft models.

\begin{tabular}{lccccccc}
\hline & \multicolumn{3}{c}{ Subrenal kidney capsule } & & \multicolumn{3}{c}{ Subcutaneous layer } \\
\cline { 2 - 3 } GOLPH3 expression & Mut-mut (n) & Mut-wt (n) & P-value & & Mut-mut (n) & Mut-wt (n) & P-value \\
\hline Low & 7 & 2 & 1 & & 4 & 2 & 0.604 \\
High & 15 & 3 & & & 8 & 2 & \\
\hline
\end{tabular}

GOLPH3, Golgi phosphoprotein 3; mut, mutation; wt, wild-type.

models successfully, but transplanting into the subrenal kidney capsule produced higher engraftment rates compared with the subcutaneous layer. It is hypothesized that the superior blood supply in the subrenal kidney capsule rather than the subcutaneous layer provides a better environment for tumor growth. Hence, to get higher engraftment rates, transplanting into the subrenal kidney capsule may be a preferential method. However, there are several advantages to the subcutaneous layer way method. Compared with the subrenal kidney capsule engraftment, shorter time and fewer procedures are required to establish xenografts in the subcutaneous layer. Therefore, if the aim is to establish large numbers of xenografts, the subcutaneous layer method may be recommended.

In addition, the present results demonstrated for the first time that high expression of GOLPH3 in the original tumor may signify better take rates in xenograft models, compared with low expression. According to previously published studies, high GOLPH3 expression promotes tumor cell proliferation (12-15). It is thus hypothesized that this increased cell proliferation may promote the high engraftment rates in xenograft models. The present results also demonstrated that most successful xenografts carried the GOLPH3 mutant and only a small portion of them lost expression. Therefore, most xenografts appeared to express the genotype and phenotype of the original tumor successfully, but fewer changes in genotype and phenotype may be observed when xenografts grow in nude mice microenvironment compared with the normal microenvironment.
In summary, the present study demonstrated that GOLPH3 may be an important diagnosis and prognosis indicator in patients with NSCLC. The genotype and phenotype of tumor xenograft models derived from patient lung cancer tissues exhibited similarities to the originating primary tumors. High expression of GOLPH3 may promote the successful establishment of xenograft models. The present results may form the basis for further studies on GOLPH3 or xenograft models in NSCLC.

\section{Acknowledgments}

Not applicable.

\section{Funding}

The present study was supported by the National Natural Science Foundation of China (grant nos. 81402306, 81760481 and 81360312), the Guangxi Natural Science Foundation Program (grant no. 2014GXNSFBA118158) and the Science and Technology Research Project of the Guangxi Colleges and Universities (grant no. YB2014080).

\section{Availability of data and materials}

All data generated or analyzed during this study are included in this published article. 
A
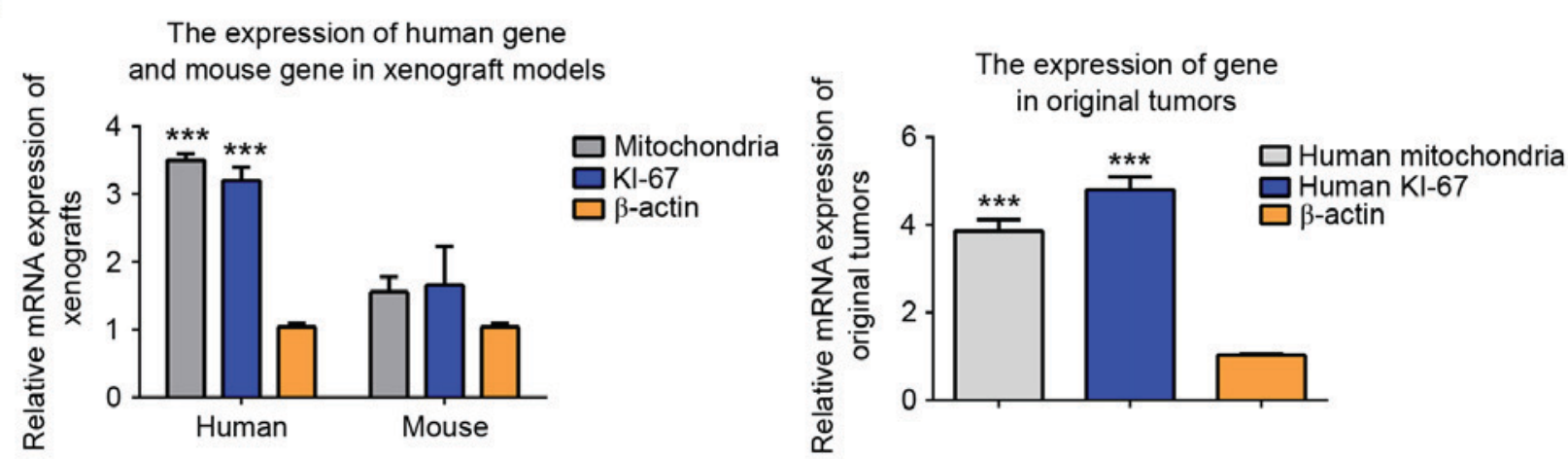

B
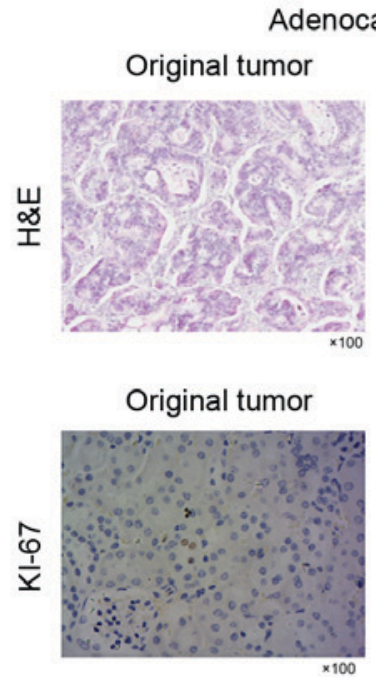

Original tumor

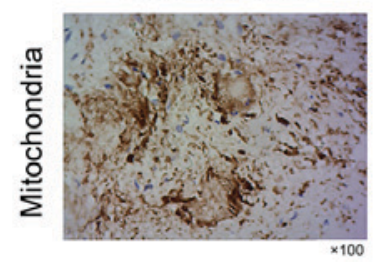

Xenograft
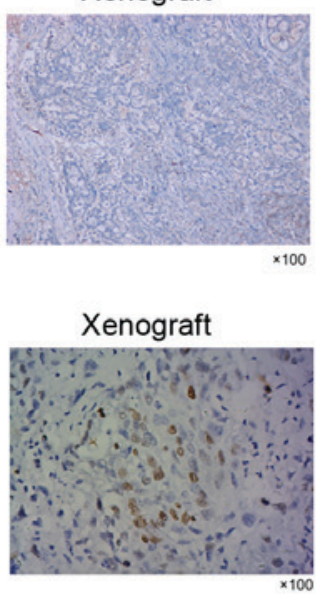

Xenograft

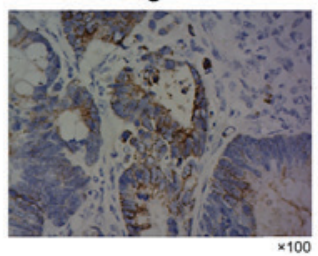

C
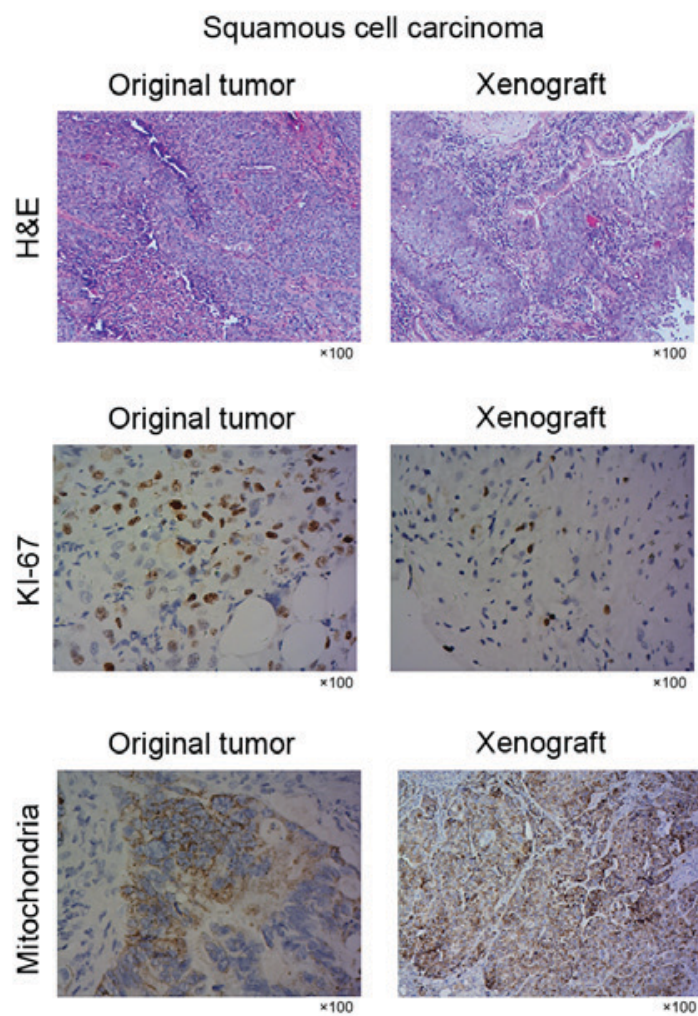

Xenograft

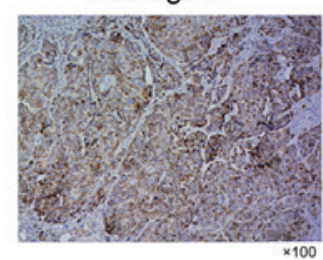

Figure 2. Confirmation of human origin of the mouse xenograft tumors. (A) mRNA expression of mitochondria (human-vs. $\beta$-actin and mouse-specific vs. $\beta$-actin, respectively) and Ki-67 (human- vs. $\beta$-actin and mouse-specific vs. $\beta$-actin, respectively) markers was measured in the mouse xenograft tumors. Mitochondria and KI-67 expressed vs. $\beta$-actin respectively in the original patient NSCLS tissues. ${ }^{* * *} \mathrm{P}<0.001$ vs. $\beta$-actin. (B) Representative images from H\&E and immunohistochemistry staining from the matching xenograft and patient tumor of a human adenocarcinoma (magnification, x100). (C) Representative images from $\mathrm{H} \& \mathrm{E}$ and immunohistochemistry staining from the matching xenograft and patient tumor of a human squamous cell carcinoma (magnification, $\mathrm{x} 100)$. NSCLC, non-small cell lung cancer.

\section{Authors' contributions}

Tumor tissues were collected by ST, XZ and HP. ST and $\mathrm{JL}$ analyzed and interpreted the patient data. ST, XZ and HP established the xenograft models. Reverse transcription-quantitative polymerase chain reaction, mutation analysis and immunohistochemistry were performed by ST, $\mathrm{XZ}$ and RY. Statistical analysis was performed by ST, JL and RY.

\section{Ethics approval and consent to participate}

The protocols of the present study were approved by the Ethics Committee of Tumor Hospital of Guangxi Medical University
(Nanning, China). The animal experiments obeyed ARRIVE Guidelines and AVMA Guidelines for the Euthanasia of Animals 2013 Edition. Written informed consent was acquired from all enrolled patients.

\section{Consent for publication}

Written informed consent was obtained from all patients for their information to be stored in the hospital database, used for research and published.

\section{Competing interests}

The authors declare that they have no competing interests. 


\section{References}

1. Barton MK: Patients of all ages with advanced non-small cell lung cancer are not receiving chemotherapy. CA Cancer J Clin 65: 337-338, 2015.

2. Torre LA, Bray F, Siegel RL, Ferlay J, Lortet-Tieulent J and Jemal A: Global cancer statistics, 2012. CA Cancer J Clin 65: 87-108, 2015

3. Siegel RL, Sahar L, Portier KM, Ward EM and Jemal A: Cancer death rates in US congressional districts. CA Cancer J Clin 65: 339-344, 2015.

4. Wu CC, Taylor RS, Lane DR, Ladinsky MS, Weisz JA and Howell KE: GMx33: A novel family of trans-Golgi proteins identified by proteomics. Traffic 1: 963-975, 2000.

5. Dippold HC, Ng MM, Farber-Katz SE, Lee SK, Kerr ML, Peterman MC, Sim R, Wiharto PA, Galbraith KA, Madhavarapu S, et al: GOLPH3 bridges phosphatidylinositol-4-phosphate and actomyosin to stretch and shape the Golgi to promote budding. Cell 139: 337-351, 2009.

6. Zhou J, Xu T, Qin R, Yan Y, Chen C, Chen Y, Yu H, Xia C, Lu Y, Ding X, et al: Overexpression of Golgi phosphoprotein-3 (GOLPH3) in glioblastoma multiforme is associated with worse prognosis. J Neurooncol 110: 195-203, 2012.

7. Abraham RT: GOLPH3 links the Golgi network to mTOR signaling and human cancer. Pigment Cell Melanoma Res 22: 378-379, 2009.

8. Scott KL, Kabbarah O, Liang MC, Ivanova E, Anagnostou V, Wu J, Dhakal S, Wu M, Chen S, Feinberg T, et al: GOLPH3 modulates mTOR signalling and rapamycin sensitivity in cancer. Nature 459: 1085-1090, 2009.

9. Buschman MD, Rahajeng J and Field SJ: GOLPH3 links the Golgi, DNA damage, and cancer. Cancer Res 75: 624-627, 2015.

10. Tang W, Han M, Ruan B, Jin W, Lou J, Yuan X, Chen D, Chen Y, Shin VY, Jin H and Wang X: Overexpression of GOLPH3 is associated with poor survival in Non-small-cell lung cancer. Am J Transl Res 8: 1756-1762, 2016.

11. Hua X, Yu L, Pan W, Huang X, Liao Z, Xian Q, Fang L and Shen H: Increased expression of Golgi phosphoprotein-3 is associated with tumor aggressiveness and poor prognosis of prostate cancer. Diagn Pathol 7: 127, 2012.

12. Feng Y, He F, Wu H, Huang H, Zhang L, Han X and Liu J: GOLPH3L is a novel prognostic biomarker for epithelial ovarian cancer. J Cancer 6: 893-900, 2015.

13. Zhu K, Zhao Q, Yue J, Shi P, Yan H, Xu X and Wang R: GOLPH3 overexpression correlates with poor response to neoadjuvant therapy and prognosis in locally advanced rectal cancer. Oncotarget 7: 68328-68338, 2016.

14. Xue Y, Wu G, Liao Y, Xiao G, Ma X, Zou X, Zhang G, Xiao R, Wang X, Liu Q, et al: GOLPH3 is a novel marker of poor prognosis and a potential therapeutic target in human renal cell carcinoma. Br J Cancer 110: 2250-2260, 2014.

15. Zeng Z, Lin H, Zhao X, Liu G, Wang X, Xu R, Chen K, Li J and Song L: Overexpression of GOLPH3 promotes proliferation and tumorigenicity in breast cancer via suppression of the FOXO1 transcription factor. Clin Cancer Res 18: 4059-4069, 2012.

16. Seol HS, Suh YA, Ryu YJ, Kim HJ, Chun SM, Na DC, Fukamachi H, Jeong SY, Choi EK and Jang SJ: A patient-derived xenograft mouse model generated from primary cultured cells recapitulates patient tumors phenotypically and genetically. J Cancer Res Clin Oncol 139: 1471-1480, 2013.
17. Cutz JC, Guan J, Bayani J, Yoshimoto M, Xue H, Sutcliffe M, English J, Flint J, LeRiche J, Yee J, et al: Establishment in severe combined immunodeficiency mice of subrenal capsule xenografts and transplantable tumor lines from a variety of primary human lung cancers: Potential models for studying tumor progression-related changes. Clin Cancer Res 12: 4043-4054, 2006.

18. Morton CL and Houghton PJ: Establishment of human tumor xenografts in immunodeficient mice. Nat Protoc 2: 247-250, 2007.

19. van Kempen LC, Ruiter DJ, van Muijen GN and Coussens LM: The tumor microenvironment: A critical determinant of neoplastic evolution. Eur J Cell Biol 82: 539-548, 2003.

20. Wang Y, Revelo MP, Sudilovsky D, Cao M, Chen WG, Goetz L, Xue H, Sadar M, Shappell SB, Cunha GR and Hayward SW: Development and characterization of efficient xenograft models for benign and malignant human prostate tissue. Prostate 64: $149-159,2005$.

21. Lee $\mathrm{CH}$, Xue H, Sutcliffe M, Gout PW, Huntsman DG, Miller DM, Gilks CB and Wang YZ: Establishment of subrenal capsule xenografts of primary human ovarian tumors in SCID mice: Potential models. Gynecol Oncol 96: 48-55, 2005.

22. Zhang XC, Zhang J, Li M, Huang XS, Yang XN, Zhong WZ, Xie L, Zhang L, Zhou M, Gavine P, et al: Establishment of patient-derived non-small cell lung cancer xenograft models with genetic aberrations within EGFR, KRAS and FGFR1: Useful tools for preclinical studies of targeted therapies. J Transl Med 11: 168, 2013.

23. Rygaard J and Povlsen CO: Heterotransplantation of a human malignant tumour to 'Nude' mice. Acta Pathol Microbiol Scand 77: 758-760, 1969.

24. Fichtner I, Rolff J, Soong R, Hoffmann J, Hammer S, Sommer A, Becker M and Merk J: Establishment of patient-derived non-small cell lung cancer xenografts as models for the identification of predictive biomarkers. Clin Cancer Res 14: 6456-6468, 2008

25. Perez-Soler R, Kemp B, Wu QP, Mao L, Gomez J, Zeleniuch-Jacquotte A, Yee H, Lee JS, Jagirdar J and Ling YH: Response and determinants of sensitivity to paclitaxel in human non-small cell lung cancer tumors heterotransplanted in nude mice. Clin Cancer Res 6: 4932-4938, 2000.

26. Nolen RS: AVMA board approves panel on euthanasia report: Updated guidelines cover more species and methods. J Am Vet Med Assoc 239: 1269, 2011.

27. Delgado-Ruiz RA, Calvo Guirado JL and Romanos GE: Bone grafting materials in critical defects in rabbit calvariae. A systematic review and quality evaluation using ARRIVE guidelines. Clin Oral Implants Res 26: 915-930, 2015.

28. Marshman E, Ottewell PD, Potten CS and Watson AJ: Caspase activation during spontaneous and radiation-induced apoptosis in the murine intestine. J Pathol 195: 285-292, 2001.

This work is licensed under a Creative Commons Attribution-NonCommercial-NoDerivatives 4.0 International (CC BY-NC-ND 4.0) License. 